\title{
Auroral signatures of flow bursts released during magnetotail reconnection at Jupiter
}

\author{
A. Radioti, ${ }^{1}$ D. Grodent, ${ }^{1}$ J.-C. Gérard, ${ }^{1}$ and B. Bonfond ${ }^{1}$ \\ Received 27 August 2009; revised 18 February 2010; accepted 2 March 2010; published 17 July 2010.
}

[1] Recent studies based on Hubble Space Telescope (HST) data reported the presence of transient polar dawn spots in the Jovian auroral region and interpreted them as signatures of internally driven magnetic reconnection in the Jovian magnetotail. Even though an association of the polar dawn spots with the reconnection process has been suggested, it has not been yet investigated which part of the process and what mechanism powers these auroral emissions. In the present study, we examine the scenario that the auroral spots are triggered by the inward moving flow bursts released during magnetic reconnection at Jupiter. We base our analysis on a model adapted from the terrestrial case, according to which moving plasma flow burst is coupled with the ionosphere by field-aligned currents, giving rise to auroral emissions. We estimate the upward field-aligned current at the flank of the flow bursts, using in-situ magnetic field measurements and we derive the auroral emitted power. We statistically study the observed emitted power of the polar dawn spots, based on HST data from 1998 to 2007, and we compare it with the emitted power derived according to the proposed scenario. Apart from the emitted power, other properties of the polar dawn spots such as their location, periodicity, duty cycle and multiplicity suggest that they are associated with the inward moving flow bursts released during magnetic reconnection in Jupiter's tail.

Citation: Radioti, A., D. Grodent, J.-C. Gérard, and B. Bonfond (2010), Auroral signatures of flow bursts released during magnetotail reconnection at Jupiter, J. Geophys. Res., 115, A07214, doi:10.1029/2009JA014844.

\section{Introduction}

[2] Jupiter's ultraviolet auroral emissions have been extensively studied over the last years [e.g., Clarke et al., 2004]. With increasing latitude the main auroral components are: the satellite footprints, the main oval and the polar emissions. The satellite footprints are magnetically connected to the positions of the moons Io, Europa and Ganymede [e.g. Bonfond et al., 2008; Grodent et al., 2009]. The main auroral oval or main emission [Grodent et al., 2003a] is associated with the ionosphere-magnetosphere coupling current system which is related to the breakdown of corotation in the middle magnetosphere and presumably maps to the equatorial plane between $\sim 15$ and $\sim 40 \mathrm{R}_{J}$ [e.g., Cowley and Bunce, 2001; Hill, 2001]. The emissions located poleward of the main oval, the polar emissions, are magnetically connected to the outer magnetosphere and possibly related to a sector of the Dungey and Vasyliūnas cycle flows [Cowley et al., 2003; Grodent et al., 2003b]. Previous analyses based on Hubble Space Telescope data revealed several isolated transient features located poleward of the main oval such as bright transient flares [Waite et al., 2001; Gérard et al., 2003], whose brightness can rise from a few $k R$ to several MR in tens of

\footnotetext{
${ }^{1}$ Laboratoire de Physique Atmosphérique et Planétaire, Institut d'Astrophysique et de Géophysique, Université de Liège, Liège, Belgium.

Copyright 2010 by the American Geophysical Union. 0148-0227/10/2009JA014844
}

seconds, and long-lived quasi-sun-aligned polar auroral filaments of brightness of $100 \mathrm{kR}$ [Nichols et al., 2009]. Several auroral features are suggested to be triggered by reconnection processes in the Jovian magnetotail. In particular, recent studies occasionally showed the presence of parallel arc structures located poleward of the main oval on the dawn sector (multiple dawn arcs [Grodent et al., 2003a]) and isolated spots appearing in the dusk-midnight sector, poleward of the main oval (nightside polar spots [Grodent et al., 2004]). Given their properties and observed location the multiple dawn arcs and the nightside polar spots were proposed to be triggered by reconnection processes in the Jovian magnetotail. A recent work reported the presence of polar dawn spots, with a characteristic re-occurrence period of 2-3 days [Radioti et al., 2008]. These observations were based on the 2007 HST data set during which data are available on a daily basis for several time intervals and allowed one to detect periodicity. Because of their periodic re-occurrence and observed location, the polar dawn spots were interpreted as auroral signatures of internally driven magnetic reconnection in the Jovian magnetotail.

[3] Vasyliūnas [1983] proposed that an internally driven major reconfiguration process takes place in the Jovian magnetotail, in analogy with the solar wind driven terrestrial substorms. During the process, mass loading of magnetic flux tubes leads to a stretched tail configuration so that at some point the magnetic tension can no longer balance the enhanced centrifugal forces and reconnection is initiated. As 
a consequence, plasmoids are released downtail and the inner part of the magnetosphere relaxes to a less stretched configuration. Plasma flow bursts are directed away and towards the planet. Galileo spacecraft detected signatures of the internally driven reconnection process concentrated beyond $60 R_{J}$ in the predawn tail region sector, occurring with a characteristic period of 2-3 days. The reconnection signatures consist of energetic particle bursts characterized by strong radial anisotropies [Woch et al., 1998; Krupp et al., 1998; Kronberg et al., 2008], intensifications in the auroral radio emissions [Louarn et al., 1998], bipolar fluctuations of the north-south and distortions of the azimuthal magnetic field component [Woch et al., 1999; Russell et al., 2000; Kronberg et al., 2005; Vogt et al., 2010], changes in the energetic particle energy spectra [Woch et al., 1998] and heavy ion composition enhancements [Radioti et al., 2007]. Additionally, recent New-Horizons observations revealed velocity dispersions, anisotropies and compositional variations in the deep Jovian magnetotail $\left(>500 \mathrm{R}_{J}\right)$ with a $\sim 3$ day periodicity $[\mathrm{McNutt}$ et al., 2007]. These observations were interpreted as signatures of energetic particle streaming away from the planet and injection sites in the near-tail region, possibly associated with magnetic reconnection events.

[4] Inward moving flow bursts [e.g., Sergeev et al., 2000; Nakamura et al., 2001; Zong et al., 2007] in the terrestrial magnetosphere are associated with auroral activations based on the concept of the bubble model [Chen and Wolf, 1993]. Plasma flow bursts released at the reconnection site become electrically polarized, because of the charge accumulation at the bubble flanks. As a result Alfvén waves are launched in a sense where upward field-aligned current is created at the duskward edge in a similar way to the current wedge [Chen and Wolf, 1993]. While several studies at Earth reported an association of the planetward flow bursts with auroral activations, such a relation has not been yet shown at Jupiter. Even though previous studies [Grodent et al., 2003a, 2004; Radioti et al., 2008] suggested that transient auroral spots are associated with the internally driven reconnection process in the Jovian magnetotail, it is still unclear which part of the process triggers the auroral emissions. In the present work we study the scenario in which polar dawn spots observed in the Jovian aurora are powered by the inward (planetward) moving flow bursts released during magnetotail reconnection.

\section{HST Observations: Emitted Power}

[5] The polar dawn spots have been initially introduced to describe the spots observed poleward of the main oval in the dawn sector whose re-occurrence has a characteristic period of 2-3 days [Radioti et al., 2008]. These observations were based only on the 2007 Hubble Space Telescope (HST) data set, during which data are available on a daily basis for several time intervals. The present study equally considers the polar dawn spots and the multiple dawn arcs [Grodent et al., 2003a], which were also associated with tail reconnection. The multiple dawn arcs consist of parallel arc structures located poleward of the main oval in the dawn sector. Their properties: location, duration, size, emitted power, fall into the same category as those of the polar dawn spots. Therefore, in the following, we use the term polar dawn spots to describe similar structures observed during the 1998-2007 data set with those initially reported based on the 2007 data set, including the multiple dawn arcs. The nightside polar spots are not systematically observed by HST, because the midnight sector is often hidden behind the planetary disk and as a result we can not conclude on their periodic re-occurrence. However, their other observed properties are similar to those reported for the polar dawn spots and multiple dawn arcs and may therefore be considered to be associated with the same magnetotail dynamics. These features are not included in the sample analyzed here, but we suggest that the triggering mechanism is similar.

[6] This work is based on FUV images acquired with the Space Telescope Imaging Spectrograph (STIS) and the Advanced Camera for Surveys (ACS) on board HST. The pixel resolution is 0.024 and 0.030 arcsec for the STIS and ACS images, respectively. We applied a dark count substraction, flat fielding and geometric correction to all the images [e.g., Grodent et al., 2003a]. Figure 1 shows a sequence of the FUV auroral emissions at the north pole of Jupiter measured by HST/ACS on 31 May 2007. The arrows in Figure 1a point to the main auroral features: main oval (main emission), Io footprint and its trail, polar emissions and polar dawn spots. Polar dawn spots are also indicated in Figures $1 \mathrm{~b}$ and 1c. On that day HST captured Jupiter's north aurora in a sequence of 18 images taken 2-3 minutes apart, between 11:41 and 12:21 UT. During this period three spots appear and two of them tend to disappear towards the end of the sequence. At the beginning of the time interval only one spot exists (Figure 1a) later a second spot appears (Figure 1b) and towards the end of the sequence a third one is observed (Figure 1c). The evolution of the emitted power of the three spots as a function of time is shown in Figure 2. The power of the two spots ('a' and ' $b$ ') reaches a peak, maximum power, which is used later on for the statistical analyses. Further in the time sequence the power of the spots tend to vanish with time while that of spot 'c' is increasing, judging from the two available sequences. Radioti et al. [2008] reported examples of spots appearing and completely disappearing.

[7] Figure 3 shows the histogram of the maximum power above the background emission of 53 polar dawn spots, measured from 1998 to $2007.55 \%$ of the data included in the histogram were obtained during 2007, when frequent observations are available. Only the cases where the spots are well separated from the rest of the emission are considered. For several cases HST did not capture the whole evolution of the spots (like spot 'c' shown in Figure 1) because of the limited time sequences. For such cases we use the white bars to denote that it is uncertain whether the measured power reached its maximum value. The histogram shows that the highest measured power reaches values of $10 \mathrm{GW}$ while the majority of the spots has a maximum emitted power of $2.5 \mathrm{GW}$. The observed emitted power is compared with the emitted power estimated on the basis of the bubble model [Chen and Wolf, 1993], in the next section.

\section{Inward Moving Flow: Estimate of the Field- Aligned Currents and Emitted Power}

[8] In this section we estimate the emitted auroral power based on the concept of the bubble model, initially introduced for the Earth [Chen and Wolf, 1993] according to which moving flow burst is coupled with the ionosphere by field 

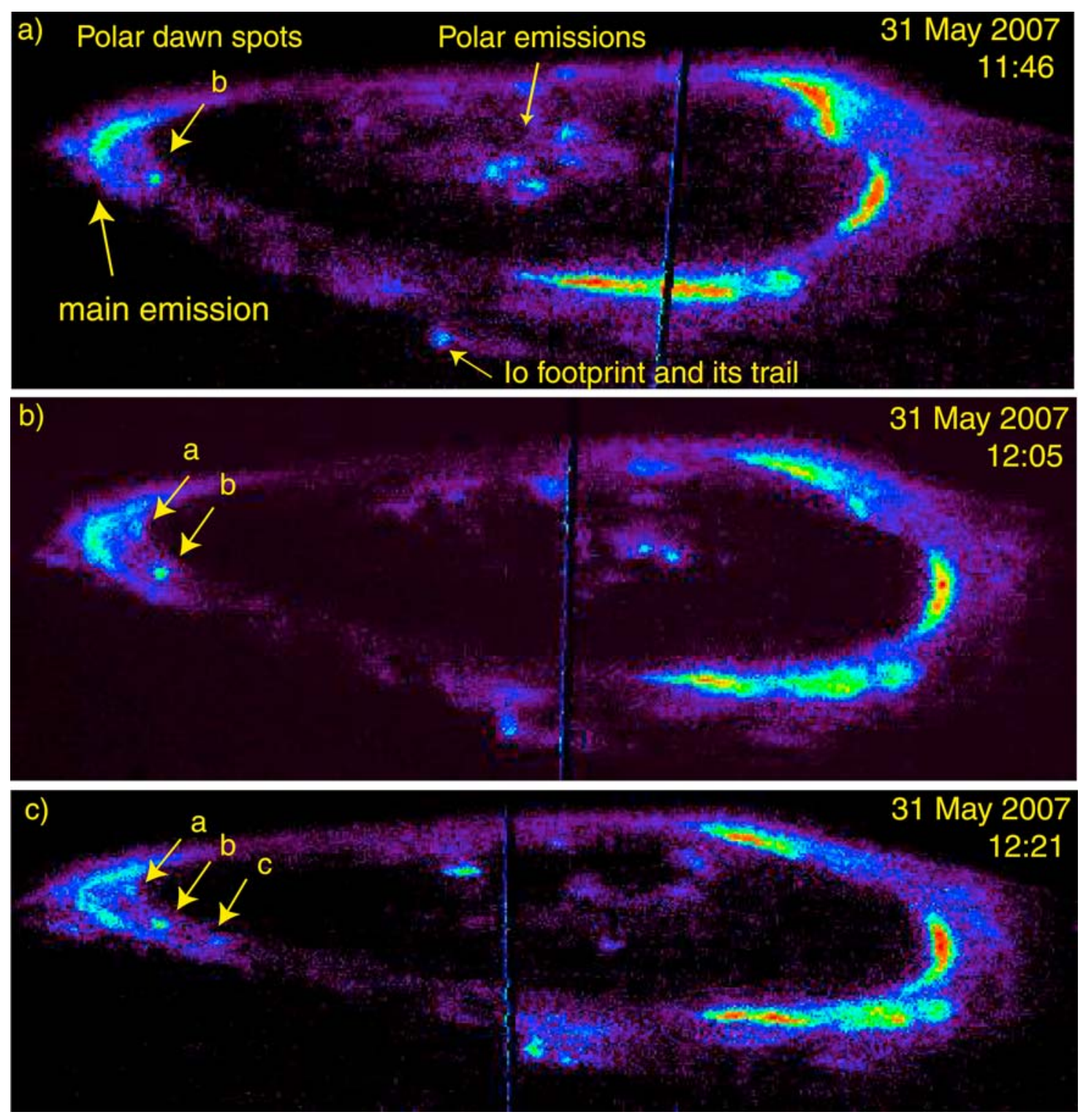

Figure 1. A sequence of three FUV auroral emissions at the north pole of Jupiter measured by HST/ACS on 31 May 2007 at (a) 11:46, (b) 12:05, and (c) 12:21 UT. The images are background subtracted assuming a Minnaert function fit of the planetary disk. The central meridian longitude in SIII is $161^{\circ}, 172.5^{\circ}$ and $182.6^{\circ}$, for each image respectively. The arrows in Figure 1a indicate the main auroral features: the main oval (main emission), the Io footprint and its trail, the polar emissions and the polar dawn spots. Polar dawn spots are also marked in Figures $1 \mathrm{~b}$ and 1c.

aligned currents, giving rise to auroral emissions. This model has been the basis of several studies in the terrestrial magnetosphere, in which inward moving flow bursts are associated with activations of auroral patches in the high-latitude region [e.g., Sergeev et al., 2000; Nakamura et al., 2001]. Here we adapt the bubble model by Chen and Wolf [1993] to the Jovian case and we show a schematic representation of the proposed mechanism in Figure 4.

[9] We make use of magnetic field measurements during inward moving flow bursts in order to quantify the current flowing at the flank of the bubble. Galileo reported on observations of flow bursts based on set of measurements consisting of ion intensity, anisotropy and magnetic field data [e.g., Krupp et al., 1998; Woch et al., 1999; Kronberg et al.,
2008; Vogt et al., 2010]. Among others, the flow bursts are characterized by distortions of the azimuthal component [Kronberg et al., 2005]. In this study we use the magnetic field azimuthal component $B_{\varphi}$ during the time interval of inward moving flow bursts, measured by Galileo on DOY 2711996 at $\sim 103 R_{J}$ and at $\sim 02: 30$ local time [Kronberg et al., 2005], shown in Figure 5b. Figure 5 shows several examples of inward moving flow bursts, which consist of ion intensity enhancements (not shown here), simultaneously with strong increases in the ion anisotropy in the radial inward direction (Figure 5a), indicative of inward moving flow bursts according to Kronberg et al. [2005]. The time intervals of the flow bursts are marked in Figure 5 with the perpendicular solid lines. Each burst event is accompanied with a distortion 


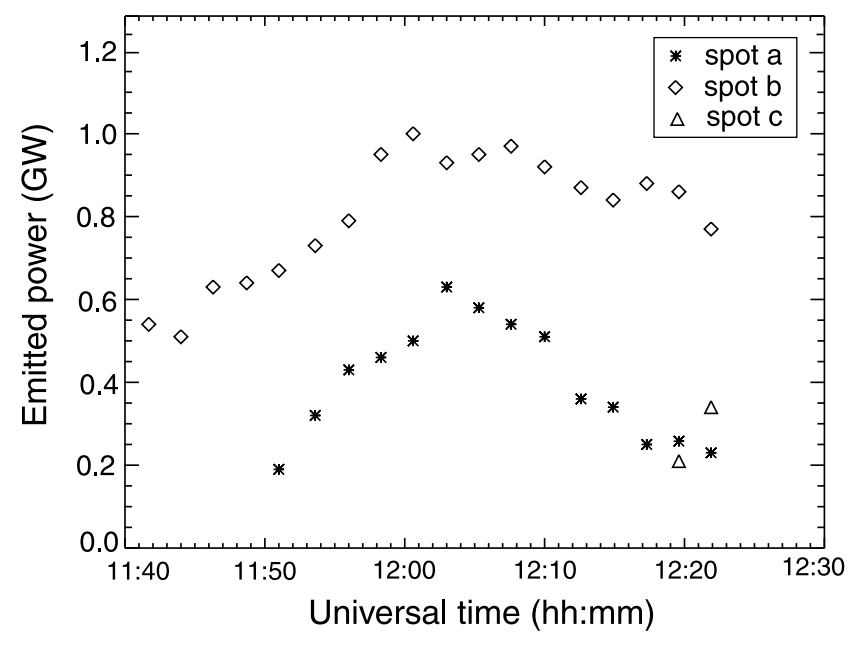

Figure 2. The emitted power of the three spots shown in Figure 1 as a function of time. The uncertainty on the measured power ranges from 0.01 to $0.02 \mathrm{GW}$.

of the azimuthal component $B_{\varphi}$, which we use to estimate the field-aligned current. In this study we consider the events, which do not fall on top of the current sheet crossings (dashed lines), in order to avoid including magnetic field signatures other than those of the field-aligned current, associated with the flow burst. The flow burst events considered in our analyses are the D ( 2 events), E and F of Figure 5. The field is changing from 2 to $-3 \mathrm{nT}, 1$ to $-3 \mathrm{nT},-3$ to $-5 \mathrm{nT}$ and -2 to $-5 \mathrm{nT}$, respectively, over $\sim 40$ minutes time interval.

[10] According to Ampère's law the observed reduction in the azimuthal component $B_{\varphi}$ is indicative of a layer of net upward-directed field-aligned currents (FACs), flowing from the ionosphere to the equator. We use Ampère's law to estimate the FAC flowing at the current loop, implied by the changes in the azimuthal magnetic field component. The changes are observed at some point between the ionosphere and the closure currents in the equatorial magnetosphere, during the flow burst. Application of Ampère's law to a circular loop passing through the point and centered on the magnetic axis assuming axisymmetry shows that the equatorward height-integrated Pedersen current per radian of azimuth, I, flowing in the ionosphere at the feet of the field lines, is given by:

$$
I=\frac{\rho B_{\varphi}}{\mu_{0}}
$$

where $\rho$ is the perpendicular distance centered on the magnetic axis to the point of the measurement and $\mu_{0}$ is the magnetic permeability of free space. The variable $\rho$ is approximately equal to the radial distance of the observation point $\left(\sim 103 R_{J}\right)$, since Galileo was close to the equatorial plane at the given time. Figure 5c (left axis) shows the Pedersen current per radian of azimuth $I$ derived by the azimuthal component $B_{\varphi}$, using equation (1). Because of current continuity the $I$ is equal to the net FAC per radian of azimuth flowing into the ionosphere between the pole and the observation point. A decrease in $I$ moving towards the equator implies that a similar amount of FACs leaves the ionosphere, while an increase of $I$ means that the same amount of FACs enters the ionosphere. Accordingly, arrows are marked on the plot (Figure 5c) to indicate the direction of the FACs identified as either upward (directed out of the ionosphere) or downward (directed into the ionosphere).

[11] We integrate the current $I$ over the azimuthal size of the flowing bubble, so as to estimate the current flowing at the flank of the bubble:

$$
I_{b u b}=\theta \frac{\rho B_{\varphi}}{\mu_{0}}
$$

where $\theta=2 \operatorname{atan} \frac{S / 2}{R}, S$ is the azimuthal extent (cross tail size extent or width) of the flowing bubble and $R$ the equatorial radial distance, where the flow burst is observed. The mean azimuthal extent of the flows is estimated by Vogt et al. [2010] to be $\sim 18.3 R_{J}$. This estimation is based on a mean azimuthal velocity $\left|V_{\varphi}\right|=236 \mathrm{~km} / \mathrm{s}$ and mean duration of the flow bursts $\sim 92$ minutes, without discriminating between inward and outward flow bursts. We take into account that the inward moving flow bursts last less than the outward and approximately $1 \pm 0.7$ hours [Kronberg et al., 2008], we use the mean azimuthal velocity $\left|V_{\varphi}\right|=236 \mathrm{~km} / \mathrm{s}$ [Vogt et al., 2010] and we derive an azimuthal extent of the bubble $\mathrm{S}=$ $10 \pm 7.5 R_{J}$. Additionally, the flow bursts considered in the present study last on average $\sim 40$ minutes, which corresponds to azimuthal extent of 7.5 $R_{J}$. Thus, in our analyses we consider indicatively the azimuthal extent of the bubble to range between 5 to $10 R_{J}$. The right axis of Figure $5 \mathrm{c}$ shows the value of $I_{b u b}$ derived from equation (2) for an azimuthal extent $S=5 R_{J}$. During the time interval of the flow burst F, for example, the FAC at the flank of the bubble $I_{b u b}$ decreases from -0.6 to $\sim 1.4 \mathrm{MA}$, meaning that a net FAC amount $\left(\Delta I_{b u b}\right)$ of $\sim 0.8 \mathrm{MA}$ leaves the ionosphere (upward current). The upward-directed FAC is carried by downward moving electrons and can trigger auroral emissions. The upward current is then followed by a downward current as the $I_{b u b}$ increases. The burst events D $(1,2), \mathrm{E}$ and $\mathrm{F}$ show that a net FAC amount of 1.4, 1.6, 0.6 and $0.8 \mathrm{MA}$ enters the ionosphere, respectively. For the estimation of the emitted power, we consider an average FAC of $\sim 1$ MA.



Figure 3. Histogram showing the maximum emitted power of 53 polar dawn spots measured by HST STIS and ACS from 1998 to 2007 . The white bars represent the cases for which it is uncertain whether the measured power reached a maximum value, mainly as a result of the sparse sampling of the data set. 


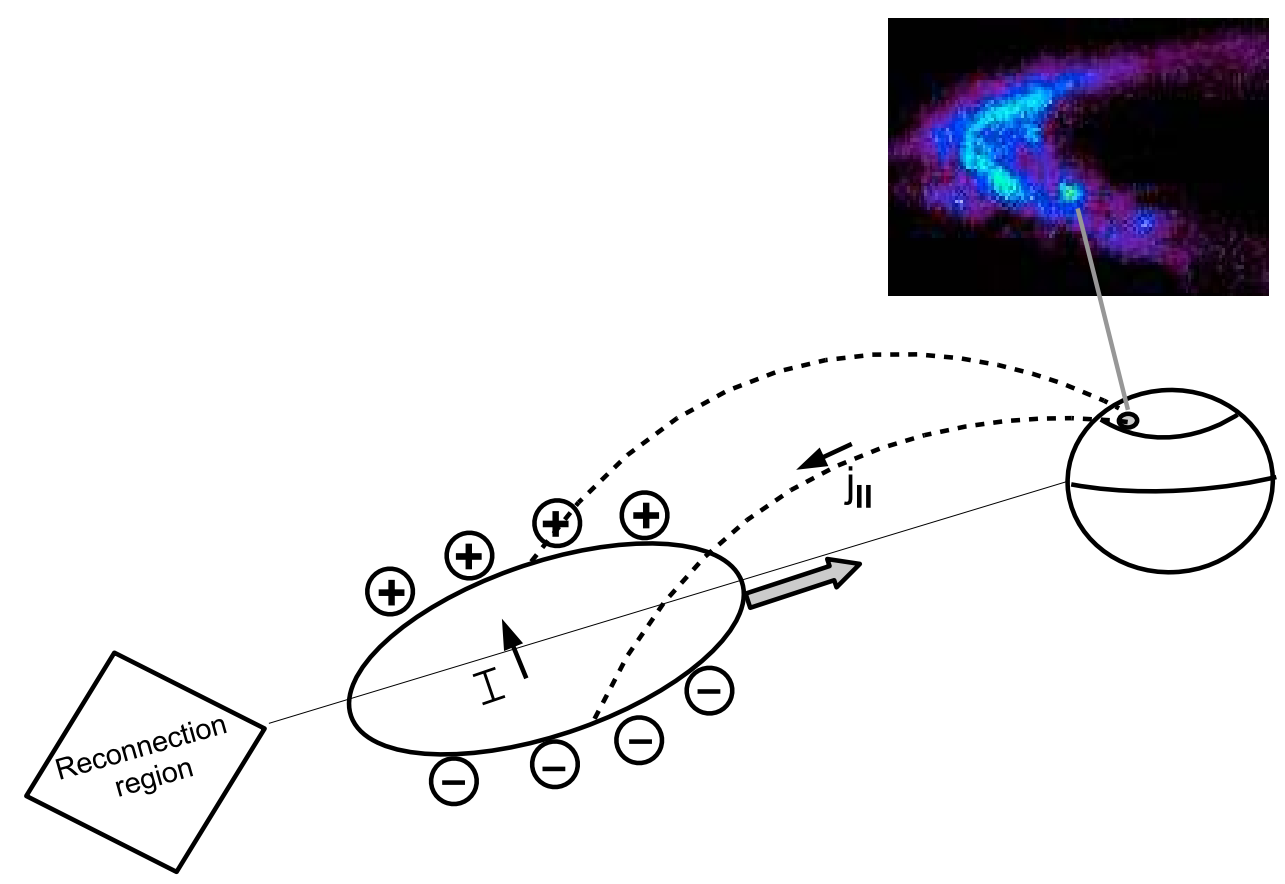

Figure 4. Sketch illustrating the relationship between auroral spots in the ionosphere and the flowing bubble released during magnetic reconnection. $I$ is the ionospheric Pedersen current, integrated over the size of the bubble (in the present analysis it is denoted as $I_{b u b}$ ). The dashed lines show the field aligned currents $j_{\mathrm{i}}$ connecting the bubble flanks with the ionosphere and the thick grey arrow indicates the direction of the flowing bubble towards the planet. The auroral spots are related to the upward field-aligned current (directed from the ionosphere to the equator) at the dawn side of the flow burst. Adapted for Jupiter from Nakamura et al. [2001].

[12] The maximum amount of power $P$ generated by a flow burst moving towards the planet is given by the voltage $V$ across the bubble multiplied by the current carried at the flank of the bubble $I_{b u b}$ :

$$
P=V \cdot I_{b u b} .
$$

The voltage across the bubble is given by $V=E \cdot S$, where $E$ is the convection electric field and $S$ the azimuthal extent of the bubble. We calculate the voltage for an average convection electric field $E \sim 2 \pm 1.25 \mathrm{mV} \mathrm{m}^{-1}$ estimated during the plasmoid release (for ion bulk velocity of $\sim 500 \mathrm{~km} / \mathrm{sec}$ and $\Delta B_{\theta} \sim 4 \mathrm{nT}$ ) [Kronberg et al., 2008]. It is significantly larger (one order of magnitude) than the ambient convection electric field $\left(0.25 \pm 0.13 \mathrm{mV} \mathrm{m}^{-1}\right)$. From the voltage $V$ across the flowing bubble and the current $I_{b u b}$ carried at its flank, we derive the available (generated) power using equation (3). However, only a fraction of the available power is dissipated in order to accelerate electrons in the Jovian ionosphere. A simple estimate showed that only $2.5 \%$ of the total available power is necessary to accelerate auroral electrons in order to produce the emitted observed power at the footprint of Ganymede in the Jovian ionosphere [Grodent et al., 2009]. Here, we assume a range of 1 to $10 \%$ of the total available power to derive the dissipated power in the ionosphere. We finally convert the injected electron energy into auroral UV emission assuming $10 \%$ efficiency for converting injected power into FUV auroral power [Grodent et al., 2001]. We follow the same analyses for an azimuthal extent of the bubble of $10 R_{J}$ and we summarize the derived values in
Table 1. The auroral emitted power based on the concept of the bubble model reaches values between 0.3 to $11 \mathrm{GW}$ (for $\mathrm{S}=5 R_{J}$ ), a similar order of magnitude of the observed maximum power shown in the histogram. For azimuthal extent of $10 R_{J}$ the predicted values of the emitted power range between 1 to $50 \mathrm{GW}$. However, such large emissions as $50 \mathrm{GW}$ are not supported by the present observations. Polar dawn spots with large emissions $(>10 \mathrm{GW})$ if any, would possible overlap with the main emission and would not be easily discriminated, and thus not included in the histogram, which considers only the polar dawn spots that are well separated from the rest of the emission.

\section{Discussion}

[13] Previous studies [Grodent et al., 2003a, 2004; Radioti et al., 2008] suggested an association of the auroral spots with magnetotail reconnection. However, it was never investigated which part of the reconnection process and what mechanism powers these auroral emissions. In the present work, we propose that the polar dawn spots, including the multiple dawn arcs, are powered by the inward moving flow bursts released during tail reconnection. We suggest that the same mechanism triggers the nightside polar spots [Grodent et al., 2004], given their similar properties with the polar dawn spots and multiple dawn arcs. The auroral emissions are considered to be associated with the inward moving flow bursts since an outward moving flow burst is disconnected from the planet. We base our analysis on a model adapted from the terrestrial case, according to which moving flow burst is coupled with 


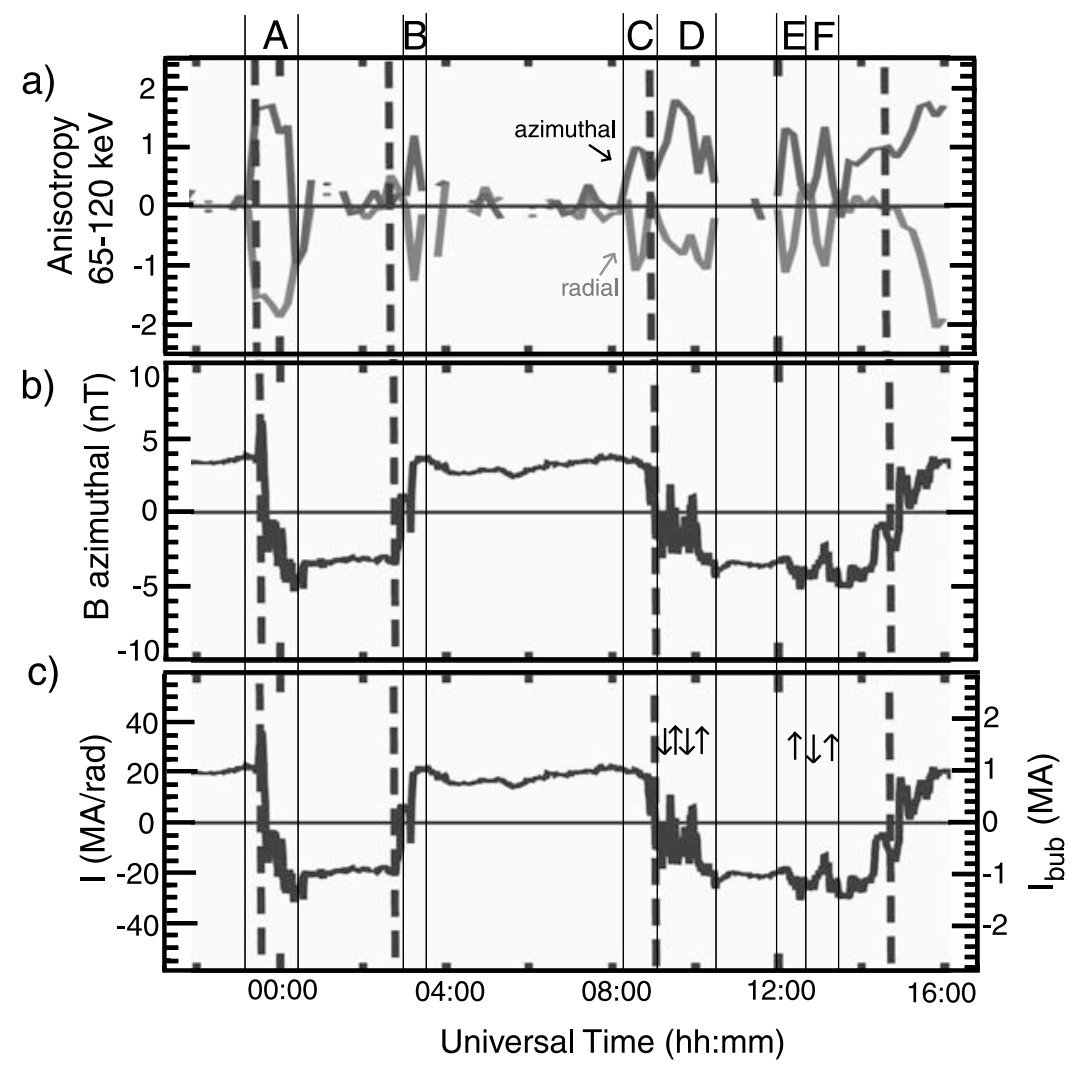

Figure 5. (a) First-order ion anisotropies in the radial and corotation direction and (b) the magnetic field azimuthal component $B_{\varphi}$ as a function of time during inward flow bursts measured by Galileo on DOY 271 1996 at $103 R_{J}$ and at $\sim 02: 30$ local time (Figures 5a and 5b are taken from Kronberg et al. [2005]). (c) Left axis shows the equatorward directed height-integrated Pedersen current per radian of azimuth $I$ derived from the $B_{\varphi}$ using equation (1). The scale on the left axis indicates the equatorward directed height-integrated Pedersen current integrated over the size of the flowing bubble $I_{b u b}$ for $5 R_{J}$ azimuthal extent of the bubble, derived from equation (2). The dashed lines show the current sheet crossings. The vertical solid lines indicate the intervals (A, B, C, D, E and F) that Galileo measures the inward moving flow bursts and the arrows the direction of the field-aligned currents.

the ionosphere by field-aligned currents, giving rise to transient auroral emissions (Figure 4). In our calculations we use in-situ magnetic field measurements during inward moving flow bursts obtained by Galileo and we estimate the emitted auroral power. Additionally, we statistically study the emitted power of the polar dawn spots, based on HST data from 1998 to 2007 , and compare it with the derived one. The estimated auroral power based on the concept of the bubble model has a similar order of magnitude with the observed maximum power of the polar dawn spots, supporting the proposed scenario.

[14] Apart from the emitted power, which constitutes the basis of the present study, there is further evidence that the auroral spots are associated with the inward moving flow bursts, such as the location of the spots. According to recent reports [Grodent et al., 2003a; Radioti et al., 2008] the spots are located poleward of the main oval close to the theoretical projected location of the Vasyliūnas-cycle tail X-line in the ionosphere, as suggested by Cowley et al. [2003]. Theoretical studies [Vasyliūnas, 1983] and in-situ observations [Woch et al., 2002] suggest that the inward moving flow is observed close to the X-line, in particular planetward of it, in accordance with the observed location of the auroral spots in the auroral region. Additionally, a statistical analysis of the polar dawn spots [Radioti et al., 2008] showed that their source region in the equatorial plane is approximately in the dawn sector, close to the observed signatures of tail reconnection, including flow bursts. However, this approach was based on the VIP4 model [Connerney et al., 1998], which is known to be inaccurate beyond the orbit of Io. An updated model, taking into account the swept-back configuration in the tail region (due to subcorotation of the plasma), would magnetically map the location of the spots earlier in local time (K. Khurana, private communication, 2009). This would

Table 1. Derived Auroral Emitted Power

\begin{tabular}{|c|c|c|}
\hline Azimuthal Extent of Bubble & $5 R_{J}$ & $10 R_{J}$ \\
\hline$\Delta I_{b u b}(\mathrm{MA})$ & 1 & 2 \\
\hline Voltage (KV) & $700 \pm 450$ & $1400 \pm 900$ \\
\hline Available power (GW) & $700 \pm 450$ & $2800 \pm 1800$ \\
\hline Dissipated power ${ }^{\mathrm{a}}(\mathrm{GW})$ & $7 \pm 4.5$ to $70 \pm 45$ & $28 \pm 18$ to $280 \pm 180$ \\
\hline Auroral emission ${ }^{\mathrm{b}}$ (GW) & $0.7 \pm 0.4$ to $7 \pm 4$ & $2.8 \pm 1.8$ to $28 \pm 18$ \\
\hline
\end{tabular}

${ }^{\mathrm{a}} 1-10 \%$ of the available power is assumed to be dissipated.

${ }^{\mathrm{b}}$ Assuming $10 \%$ efficiency for converting injected power into FUV auroral power [Grodent et al., 2001]. 
place the source region of the polar dawn spots in the predawn to midnight equatorial sector, directly on the region of the observed signatures of tail reconnection.

[15] Additionally, the periodicity and the timescales of the polar dawn spots are similar to those of the observed flow burst events released during magnetotail reconnection. The polar dawn spots are observed to re-occur with a characteristic period of 2-3 days (during the 2007 data set, when daily observations were available) and the intervals during which they are observed can last up to 29 hours [Radioti et al., 2008]. Similarly, in the scheme of the internally driven reconnection process at Jupiter's magnetotail, flow burst signatures are observed to occur with a repetition period of 2-3 days [Krupp et al., 1998] and the configuration that favors reconnection is observed to last between 10 to 30 hours [Woch et al., 1999; Kronberg et al., 2005]. Additionally, long events are reported to last up to two Jovian rotations during which several short duration flow bursts are reported [Krupp et al., 1998], justifying the observed multiplicity of the polar dawn spots (see for example Figure 1). The lifetime of the polar dawn spots is estimated to range between 10 minutes to 1 hour [Radioti et al., 2008], based on measurements where the whole evolution of the polar dawn spots is observed. However, one should note that this duration could be longer than the measured, given the fact that several HST sequences, where polar dawn spots are observed, are interrupted. Additionally, emissions under the HST threshold are not taken into account. An extrapolation of the evolution of the emitted power (Figure 1) indicates that the spots last a few tens of minutes longer than what is actually measured. Finally, auroral dissipation is expected to shorten the lifetime of the inward moving flow bursts. However, the lifetime of the polar dawn spots could not be directly compared with the duration of the inward flow bursts, as observed by Galileo. The duration measured by Galileo corresponds to the time interval the spacecraft crosses the flow burst and not to its actual lifetime.

[16] The ionospheric signature of the flow bursts at Earth, the auroral streamers, appear both in the dawn and dusk auroral sectors, they are elongated in the north-south direction and they expand equatorward with time as a result of the planetward motion of the flow bursts [e.g., Nakamura et al., 2001]. The Jovian counterpart of the flow bursts (polar dawn spots and midnight spots) are observed in the dawn and midnight auroral sectors, they do not seem to be elongated in the north-south direction and an equatorward motion is not apparent at the given HST data set. The differences between the Jovian and terrestrial ionospheric counterpart of the flow bursts might be the result of the tail magnetic field configuration at Jupiter. The location of the tail reconnection line and the configuration of the field lines in the magnetosphere of Jupiter might confine the ionospheric counterpart of the flow bursts to the dawn and midnight auroral sectors, as it is discussed earlier in this section. Additionally, the magnetic field lines at Jupiter's tail are highly stretched so that a large area in the tail equatorial plane would be mapped into a narrow auroral region, affecting also the shape of the emission. Moreover, even if polar dawn spots were present in the dusk auroral sector, they would not be easily identified. The dusk aurora at Jupiter consists of diffuse auroral emissions [Radioti et al., 2009], and various highly dynamic polar features [Grodent et al., 2003b]. Thus, transient aurora, such as the polar dawn spots, rather small in size, would not be easily discriminated from the rest of the emissions. Additionally, a planetward motion of the flow bursts, as a result of the tail magnetic mapping, would possibly be negligible in the ionospheric projection, and not visible by the current HST instrumentation. Here, we briefly address and discuss some of the main differences of the ionospheric signatures of flow bursts at Jupiter and Earth. However, such a comparison deserves a more detailed analyses, which exceeds the goals of this study and needs to be extensively addressed in a future work.

\section{Summary and Conclusions}

[17] In summary, by the present study we suggest that the polar dawn spots are associated with the inward moving flow released during magnetic reconnection in the Jovian magnetotail, based on the bubble model [Chen and Wolf, 1993] initially introduced for the Earth and adapted here for the case of Jupiter. The emitted auroral power estimated by this process has the same order of magnitude with the observed maximum power of the polar dawn spots. The observed location of the auroral spots in the ionosphere and their magnetically mapped location in the equatorial plane matches the one of the inward moving flow bursts, based on observations and theoretical studies. Finally, the periodicity, the duty cycle and the multiplicity of the polar dawn spots are in accordance with the observed properties of the inward moving flow bursts. All the above evidence confirms previous indications that the polar dawn spots are auroral signatures of magnetotail reconnection and particularly suggest their association with the inward moving flow bursts released during the reconnection process in Jupiter's magnetotail.

[18] Acknowledgments. This work is based on observations with the NASA/ESA Hubble Space Telescope, obtained at the Space Telescope Science Institute (STScI), which is operated by AURA, inc. for NASA under contract NAS5-26555. A.R., D.G., J.-C.G. and B.B. are supported by the Belgian Fund for Scientific Research (FNRS) and the PRODEX Programme managed by the European Space Agency in collaboration with the Belgian Federal Science Policy Office.

[19] Wolfgang Baumjohann thanks Olaf Amm and another reviewer for their assistance in evaluating this paper.

\section{References}

Bonfond, B., D. Grodent, J.-C. Gérard, A. Radioti, J. Saur, and S. Jacobsen (2008), UV Io footprint leading spot: A key feature for understanding the UV Io footprint multiplicity?, Geophys. Res. Lett., 35, L05107, doi:10.1029/2007GL032418.

Chen, C. X., and R. A. Wolf (1993), Interpretation of high-speed flows in the plasma sheet, J. Geophys. Res., 98, 21,409-21,419.

Clarke, J. T., D. Grodent, S. W. H. Cowley, E. J. Bunce, P. Zarka, J. E. P. Connerney, and T. Satoh (2004), Jupiter's aurora, in Jupiter. The Planet, Satellites and Magnetosphere, edited by F. Bagenal, T. E. Dowling, and W. B. McKinnon, pp. 639-670, Cambridge Univ. Press, Cambridge, U. K.

Connerney, J. E. P., M. H. Acuña, N. F. Ness, and T. Satoh (1998), New models of Jupiter's magnetic field constrained by the Io flux tube footprint, J. Geophys. Res., 103, 11,929-11,939.

Cowley, S. W. H., and E. J. Bunce (2001), Origin of the main auroral oval in Jupiter's coupled magnetosphere-ionosphere system, Planet. Space Sci., 49, 1067-1088.

Cowley, S. W. H., E. J. Bunce, T. S. Stallard, and S. Miller (2003), Jupiter's polar ionospheric flows: Theoretical interpretation, Geophys. Res. Lett., 30(5), 1220, doi:10.1029/2002GL016030.

Gérard, J.-C., J. Gustin, D. Grodent, J. T. Clarke, and A. Grard (2003), Spectral observations of transient features in the FUV Jovian polar aurora, J. Geophys. Res., 108(A8), 1319, doi:10.1029/2003JA009901. 
Grodent, D., J. H. Waite Jr., and J.-C. Gérard (2001), A self-consistent model of the Jovian auroral thermal structure, J. Geophys. Res., 106, $12,933-12,952$.

Grodent, D., J. T. Clarke, J. Kim, J. H. Waite Jr., and S. W. H. Cowley (2003a), Jupiter's main auroral oval observed with HST-STIS, J. Geophys. Res., 108(A11), 1389, doi:10.1029/2003JA009921.

Grodent, D., J. T. Clarke, J. H. Waite Jr., S. W. H. Cowley, J.-C. Gérard, and J. Kim (2003b), Jupiter's polar auroral emissions, J. Geophys. Res. 108(A10), 1366, doi:10.1029/2003JA010017.

Grodent, D., J.-C. Gérard, J. T. Clarke, G. R. Gladstone, and J. H. Waite Jr. (2004), A possible auroral signature of magnetotail reconnection process on Jupiter, J. Geophys. Res., 109, A05201, doi:10.1029/2003JA010341.

Grodent, D., B. Bonfond, A. Radioti, J.-C. Gérard, X. Jia, J. D. Nichols, and J. T. Clarke (2009), The auroral footprint of Ganymede, J. Geophys. Res., 114, A07212, doi:10.1029/2009JA014289.

Hill, T. W. (2001), The Jovian auroral oval, J. Geophys. Res., 106 8101-8107.

Kronberg, E. A., J. Woch, N. Krupp, A. Lagg, K. K. Khurana, and K.-H Glassmeier (2005), Mass release at Jupiter: Substorm-like processes in the Jovian magnetotail, J. Geophys. Res., 110, A03211, doi:10.1029/ 2004JA010777.

Kronberg, E. A., J. Woch, N. Krupp, and A. Lagg (2008), Mass release process in the Jovian magnetosphere: Statistics on particle burst parameters, J. Geophys. Res., 113, A10202, doi:10.1029/2008JA013332.

Krupp, N., J. Woch, A. Lagg, B. Wilken, S. Livi, and D. J. Williams (1998), Energetic particle bursts in the predawn Jovian magnetotail, Geophys. Res. Lett., 25, 1249-1252.

Louarn, P., A. Roux, S. Perraut, W. Kurth, and D. Gurnett (1998), A study of the large-scale dynamics of the Jovian magnetosphere using the Galileo plasma wave experiment, Geophys. Res. Lett., 25, 2905-2908.

McNutt, R. L., Jr., et al. (2007), Energetic particles in the Jovian magnetotail, Science, 318, 220-222, doi:10.1126/science.1148025.

Nakamura, R., W. Baumjohann, R. Schödel, M. Brittnacher, V. A. Sergeev, M. Kubyshkina, T. Mukai, and K. Liou (2001), Earthward flow bursts, auroral streamers, and small expansions, J. Geophys. Res., 106 10,791-10,802.

Nichols, J. D., J. T. Clarke, J. C. Gérard, and D. Grodent (2009), Observations of Jovian polar auroral filaments, Geophys. Res. Lett., 36, L08101, doi:10.1029/2009GL037578.

Radioti, A., J. Woch, E. A. Kronberg, N. Krupp, A. Lagg, K.-H. Glassmeier, and M. K. Dougherty (2007), Energetic ion composition during reconfiguration events in the Jovian magnetotail, J. Geophys. Res., 112, A06221, doi:10.1029/2006JA012047.
Radioti, A., D. Grodent, J.-C. Gérard, B. Bonfond, and J. T. Clarke (2008), Auroral polar dawn spots: Signatures of internally driven reconnection processes at Jupiter's magnetotail, Geophys. Res. Lett., 35, L03104, doi:10.1029/2007GL032460.

Radioti, A., A. T. Tomás, D. Grodent, J.-C. Gérard, J. Gustin, B. Bonford, N. Krupp, J. Woch, and J. D. Menietti (2009), Equatorward diffuse auroral emissions at Jupiter: Simultaneous HST and Galileo observations, Geophys. Res. Lett., 36, L07101, doi:10.1029/2009GL037857. (Correction, Geophys. Res. Lett., 36, L09103, doi:10.1029/2009GL038676, 2009$.

Russell, C. T., K. K. Khurana, M. G. Kivelson, and D. E. Huddleston (2000), Substorms at Jupiter: Galileo observations of transient reconnection in the near tail, Adv. Space Res., 26, 1499-1504.

Sergeev, V. A., et al. (2000), Multiple-spacecraft observation of a narrow transient plasma jet in the Earth's plasma sheet, Geophys. Res. Lett., 27, 851-854, doi:0094-8276/00/1999GL000729.

Vasyliūnas, V. M. (1983), Plasma distribution and flow, in Physics of the Jovian Magnetosphere, edited by A. Dessler, pp. 395-453, Cambridge Univ. Press, New York

Vogt, M. F., M. G. Kivelson, K. Khurana, S. P. Joy, and R. J. Walker (2010), Reconnection and flows in the Jovian magnetotail as inferred from magnetometer observations, J. Geophys. Res., 115, A06219, doi:10.1029/2009JA015098.

Waite, J. H., Jr., et al. (2001), An auroral flare at Jupiter, Nature, 410, 787-789.

Woch, J., N. Krupp, A. Lagg, B. Wilken, S. Livi, and D. J. Williams (1998), Quasi periodic modulations of the Jovian magnetotail, Geophys. Res. Lett., 25, 1253-1256.

Woch, J., et al. (1999), Plasma sheet dynamics in the Jovian magnetotail: Signatures for substorm-like processes?, J. Geophys. Res., 26 , 2137-2140.

Woch, J., N. Krupp, and A. Lagg (2002), Particle bursts in the Jovian magnetosphere: Evidence for a near-Jupiter neutral line, Geophys. Res. Lett. 29(7), 1138, doi:10.1029/2001GL014080.

Zong, Q.-G., et al. (2007), Earthward flowing plasmoid: Structure and its related ionospheric signature, J. Geophys. Res., 112, A07203, doi:10.1029/2006JA012112.

B. Bonfond, J.-C. Gérard, D. Grodent, and A. Radioti, Laboratoire de Physique Atmosphérique et Planétaire, Institut d'Astrophysique et de Géophysique, Université de Liège, B-4000 Liège, Belgium. (a.radioti@ ulg.ac.be) 